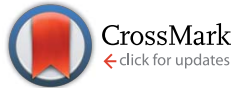

Cite this: Chem. Sci., 2017, 8, 131

Received 26th April 2016 Accepted 28th July 2016

DOI: $10.1039 / \mathrm{c} 6 \mathrm{sc} 01833 \mathrm{e}$

www.rsc.org/chemicalscience

\section{A cooperative-binding split aptamer assay for rapid, specific and ultra-sensitive fluorescence detection of cocaine in saliva $\uparrow$}

\begin{abstract}
Haixiang Yu, ${ }^{a}$ Juan Canoura, ${ }^{a}$ Bhargav Guntupalli, ${ }^{a}$ Xinhui Lou ${ }^{b}$ and Yi Xiao*a
Sensors employing split aptamers that reassemble in the presence of a target can achieve excellent specificity, but the accompanying reduction of target affinity mitigates any overall gains in sensitivity. We for the first time have developed a split aptamer that achieves enhanced target-binding affinity through cooperative binding. We have generated a split cocaine-binding aptamer that incorporates two binding domains, such that target binding at one domain greatly increases the affinity of the second domain. We experimentally demonstrate that the resulting cooperative-binding split aptamer (CBSA) exhibits higher target binding affinity and is far more responsive in terms of target-induced aptamer assembly compared to the single-domain parent split aptamer (PSA) from which it was derived. We further confirm that the target-binding affinity of our CBSA can be affected by the cooperativity of its binding domains and the intrinsic affinity of its PSA. To the best of our knowledge, CBSA-5335 has the highest cocaine affinity of any split aptamer described to date. The CBSA-based assay also demonstrates excellent performance in target detection in complex samples. Using this CBSA, we achieved specific, ultra-sensitive, one-step fluorescence detection of cocaine within fifteen minutes at concentrations as low as $50 \mathrm{nM}$ in $10 \%$ saliva without signal amplification. This limit of detection meets the standards recommended by the European Union's Driving under the Influence of Drugs, Alcohol and Medicines program. Our assay also demonstrates excellent reproducibility of results, confirming that this CBSA-platform represents a robust and sensitive means for cocaine detection in actual clinical samples.
\end{abstract}

\section{Introduction}

Aptamers are single-stranded oligonucleotides isolated via in vitro systematic evolution of ligands by exponential enrichment (SELEX), ${ }^{1,2}$ which can specifically bind to a wide range of targets including proteins, small molecules and metal ions. ${ }^{3}$ Aptamers

${ }^{a}$ Department of Chemistry and Biochemistry, Florida International University, 11200 SW 8th Street, Miami, FL, 33199, USA. E-mail: yxiao2@fiu.edu

${ }^{b}$ Department of Chemistry, Capital Normal University, Xisanhuan North Rd. 105, Beijing, 100048, China

$\dagger$ Electronic supplementary information (ESI) available: Optimization of $\mathrm{Mg}^{2+}$ and ATMND concentrations for our CBSA-based ATMND-binding assay; ATMND-reported calibration curve for CBSA-5325 at various cocaine concentrations; ATMND binding affinity for the cocaine-assembled CBSA-5325; $K_{\mathrm{D}}$ of 38-GC and different 38-GC mutants for cocaine as characterized by ITC; stem length effects on cocaine-induced CBSA assembly; spectra of CBSA-5335-based fluorescence detection of cocaine in $1 \times$ binding buffer; characterization of cocaine binding affinity of CBSA-5335 and PSA using ITC; fluorescence detection of cocaine in saliva with our fluorophore/quencher modified CBSA-5335; calibration curve of our CBSA-5335-based fluorophore/quencher assay in $1 \times$ binding buffer and $10 \%$ saliva at cocaine concentrations ranging from 0 to $10 \mu \mathrm{M}$; bias and precision of the CBSA-5335-based fluorophore/quencher assay; comparison of amplification-free split-aptamer assays for cocaine detection; sequence ID and DNA sequences used in this work. See DOI: 10.1039/c6sc01833e offer several advantages as recognition elements for biosensor applications relative to antibodies. First, aptamers can be chemically synthesized with high reproducibility at relatively low cost. ${ }^{4,5}$ Second, the high chemical stability of DNA aptamers means that they can be used under harsher conditions and stored with a longer shelf life ${ }^{6}$ Finally, it is possible to generate unstructured aptamers that form specific secondary structures such as three-way junctions ${ }^{7,8}$ or tertiary folds such as G-quadruplex structures ${ }^{\mathbf{9}, 10}$ upon target binding. Such target-induced conformational changes can be readily exploited for specific target detection in a variety of applications, including medical diagnostics, environmental monitoring and drug screening. ${ }^{\mathbf{1 1 - 1 3}}$

However, target-induced conformational change is hard to control, especially for small-molecule-binding aptamers that have relatively high dissociation constants $\left(\sim \mu \mathrm{M} K_{\mathrm{D}}\right) \cdot{ }^{\mathbf{1 4}}$ For example, the well-characterized cocaine-binding aptamer MNS$4.1\left(K_{\mathrm{D}} \sim 5 \mu \mathrm{M}\right)$ is structurally stable and forms a three-way junction even before binding cocaine..$^{15}$ To achieve an effective target-induced conformational change, Stojanovic et al. truncated the sequence to destabilize the aptamer, so that it exists in an equilibrium state consisting of both folded and unfolded structures. $^{7}$ This aptamer exhibited cocaine-induced folding, but still retained some folding activity in the absence of target, resulting in a high background that significantly limited sensor 
sensitivity. ${ }^{7,16}$ This background can be reduced by splitting the aptamer into two ${ }^{17}$ or three ${ }^{18}$ fragments, which further destabilizes the aptamer such that the fragments are unable to assemble without target. This results in minimal background signal, ${ }^{17,18}$ while the fragments retain their capacity for target recognition and can successfully reassemble into a complex secondary structure in the presence of cocaine. However, aptamer splitting results in notably reduced target affinity. ${ }^{\mathbf{1 7}}$ Thus, this approach still compromises the sensitivity of split aptamer-based sensors.

To improve the sensor's sensitivity, Zhang et al. reported an assay based on an enzyme-linked split aptamer to perform colorimetric cocaine detection. ${ }^{19}$ One of the fragments was conjugated to a plastic surface; in the presence of cocaine, this fragment would form a complex with the second fragment, which was modified with biotin in order to bind streptavidinlinked horseradish peroxidase for signal amplification. However, washing caused the dissociation of a subset of the assembled tripartite complexes, resulting in a limit of detection (LOD) of just $2.8 \mu \mathrm{M}$. To address this, Heemstra et al. incorporated a proximity ligation strategy into this enzyme-amplified split-aptamer-based assay. ${ }^{20}$ Specifically, cocaine binding facilitated the assembly of the two azide- and cyclooctyne-modified split fragments, bringing these two chemical groups into close proximity such that covalent bonds could be formed. This prevented dissociation of the assembled target-aptamer complex during washing and improved the sensor's detection performance. Although the signal was amplified by the enzyme, the sensitivity remained limited to $0.1 \mu \mathrm{M}^{21}$ due to the low binding affinity of the split aptamer $\left(K_{\mathrm{D}}=\sim 200 \mu \mathrm{M}\right) .{ }^{17}$ To overcome this limitation, we have developed a cooperative binding-based approach to generate split aptamers that retain high target affinity. Cooperative binding behavior is commonly observed in ligand-binding proteins that are highly responsive to ligand concentration, such as hemoglobin, ${ }^{22}$ ion channels ${ }^{23}$ and transcription factors. ${ }^{24}$ Those proteins generally have more than one ligand-binding site, where binding at one site increases the affinity of the other sites, resulting in a 'switch-like' binding curve. ${ }^{25}$ Breaker et al. initially found that some tandem riboswitches $^{26,27}$ naturally employ such cooperative binding ${ }^{26}$ to control gene expression in response to subtle changes in ligand concentration. This cooperative behavior was further extended into artificial biosystems such as ribozymes, ${ }^{28}$ molecular beacons ${ }^{29}$ and DNA aptamers. ${ }^{30}$ Specifically, Plaxco et al. introduced the disorder into the parent aptamer to achieve cooperative binding, and focused on the demonstration of the "switchlike" response behavior of cooperative binding that occurred when ligand concentration approaches $K_{1 / 2}\left(K_{1 / 2}\right.$ represents the ligand concentration at which half of the binding domains are occupied). These engineered cooperative DNA aptamers could not be employed for a practical sensor platform because the introduction of cooperativity unavoidably reduced the targetbinding affinity of the resulting cooperative aptamer $\left(K_{1 / 2}=\right.$ $\sim 3 \mathrm{mM}){ }^{30}$

We have for the first time successfully incorporated two tandem target-binding domains into a split aptamer (termed cooperative binding split aptamer, CBSA) to achieve more sensitive detection of cocaine, even in complex biological samples. The initial cocaine-binding event stabilizes the structure of the split aptamer and facilitates subsequent targetbinding at the second binding domain. Our CBSA exhibits higher target affinity and far more responsive target-induced aptamer assembly compared to the single-domain parent split aptamer (PSA) from which it was derived. Using a fluorophore/ quencher pair, we have demonstrated that a CBSA-based fluorescence assay can achieve sensitive and reproducible cocaine detection in biofluid specimens, with a LOD of $50 \mathrm{nM}$ in $10 \%$ saliva within $15 \mathrm{~min}$. Given the simplicity of splitting and engineering a CBSA from an isolated aptamer and the excellent performance of the CBSA-based assay in biofluid samples, it should be straightforward to develop other CBSA-based assays from existing or future aptamers for rapid, sensitive and specific detection of various targets in clinical or field settings in a simple, low-cost assay format.

\section{Results and discussion}

Cooperative target binding of split aptamers requires the incorporation of at least two binding domains into a single pair of fragments. We previously reported a cocaine-binding aptamer (38-GC) ${ }^{31}$ that exhibits 2.5 -fold higher cocaine affinity than the MNS-4.1 aptamer from which it was derived. 38-GC contains a three-way junction with the target-binding domain located at its center, surrounded by three double-stranded stems (stems 1, 2 and 3) and two loops (GAA and AAA loops). We have determined that stem 3 is essential for cocaine binding, while both stem 1 and stem 2 contribute to the stability of the three-way junction structure that forms upon target binding. ${ }^{31}$ We derived two different pairs of split aptamer fragments from 38-GC (Fig. 1A and B), in which stem 3 remained intact but the 3 '-end of stems 1 and 2 was truncated and the AAA loop of stem 2 was eliminated. We subsequently produced a construct from these two sets of split aptamers, in which stem 1 from one set was linked to stem 2 from the second set (Fig. 1C). The resulting CBSA consists of a short fragment (SF) and a long fragment (LF).

We anticipated that the CBSA fragments would remain separated in the absence of target, but would form two tandem cocaine-binding domains when fully assembled with cocaine. To confirm effective target-induced CBSA assembly, we developed a binding assay based on the fluorescent molecule 2amino-5,6,7-trimethyl-1,8-naphthyridine (ATMND). It has been reported that ATMND can strongly bind to a thymine situated opposite a C3 spacer abasic site (AP site) within a DNA duplex $\left(K_{\mathrm{D}}=111 \mathrm{nM}\right)$ via three-point hydrogen bonding. ${ }^{32}$ Although ATMND fluoresces brightly when free in solution, this fluorescence is greatly quenched when ATMND is bound to a DNA duplex in this fashion. ${ }^{33}$ We therefore replaced the adenosine (at position 10 from $5^{\prime}$ ) between the two binding domains of the short fragment with a C3 spacer to form an AP site with a thymine in the opposite position within the long fragment upon cocaine binding (Fig. 2A). In the absence of cocaine, the long and short fragments remain separated, with strong fluorescence produced by the free ATMND molecules (Fig. 2B, left). Upon addition of cocaine, the CBSA fragments undergo 


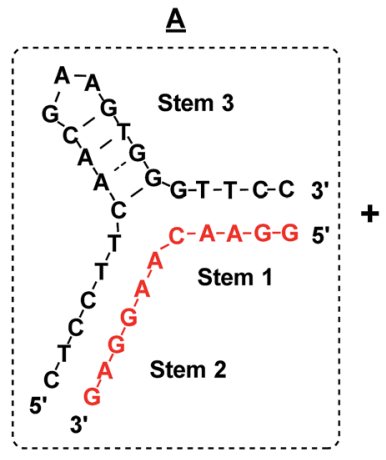

Split Cocaine-Binding Aptamers
$\underline{B}$

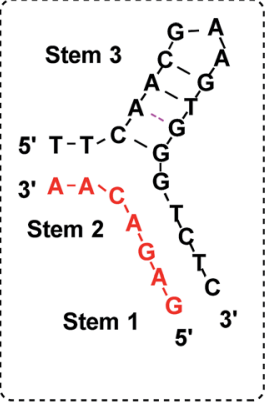

$\underline{\mathbf{C}}$

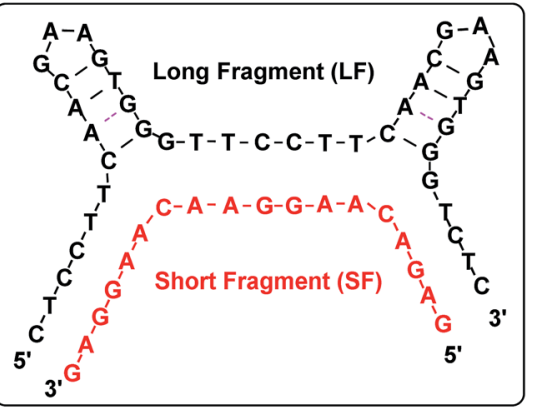

Cooperative Binding Split Aptamer (CBSA)

Fig. 1 The design of our cocaine-binding CBSA. Two split aptamer pairs were derived from 38-GC. Stem 1 of one split aptamer (A) was merged with stem 2 of the other split aptamer (B) to form an engineered CBSA (C) comprising a short fragment (SF) and a long fragment (LF).

cooperative target-induced assembly and form a duplexed AP site that binds ATMND and quenches its fluorescence (Fig. 2B, right). The resulting CBSA-5325/cocaine complex contains four complementary base-paired segments (Fig. 2A, right; segments labeled (A)-(D)) and a dinucleotide bulge formed within each three-way junction. ${ }^{34}$

We subsequently confirmed the cocaine-induced assembly of CBSA experimentally. When we mixed $1 \mu \mathrm{M}$ each of the long and short fragments with $200 \mathrm{nM}$ ATMND in $1 \times$ binding buffer (10 mM Tris- $\mathrm{HCl}, 100 \mu \mathrm{M} \mathrm{MgCl}_{2}, \mathrm{pH}$ 7.4), we observed $10 \%$ background quenching (Fig. 2C, before cocaine addition). This quenching is most likely attributable to low levels of nonspecific quench and non-target assembly of the CBSA. Upon addition of $250 \mu \mathrm{M}$ cocaine, $72 \%$ of the ATMND fluorescence was quenched within $15 \mathrm{~min}$, indicating rapid target-induced CBSA assembly (Fig. 2C, after cocaine addition). We also tested the performance of the AP-incorporating parent split aptamer (PSA) from which the CBSA was initially derived (Fig. 2D, PSA), which features only a single cocaine-binding domain, in our ATMND-binding assay. In the absence of cocaine, the PSA quenched $6 \%$ of ATMND fluorescence (Fig. 2C, before cocaine addition), and no measurable signal change was observed upon addition of $250 \mu \mathrm{M}$ cocaine (Fig. 2C, after cocaine addition). This indicates that no cocaine-induced assembly is taking place, presumably because the shortening of stem 1 to four base-pairs with an abasic site in the middle results in poor thermodynamic stability of the cocaine-PSA complex at room temperature. We therefore conclude that the quenching observed most likely arises through non-specific interactions between PSA and ATMND.

Aptamer binding affinity ${ }^{7,35}$ and DNA hybridization efficiency $^{36}$ are both strongly affected by magnesium concentration. We therefore used our ATMND-binding assay to optimize cocaine-induced CBSA assembly by varying the $\mathrm{Mg}^{2+}$ concentration from 0 to $1000 \mu \mathrm{M}$, and observed that maximum cocaine-induced ATMND quenching occurred in the presence of $100 \mu \mathrm{M} \mathrm{Mg}^{2+}$ (ESI, Fig. S1A $\dagger$ ). In the absence of cocaine, we observed $9 \%$ quenching without $\mathrm{Mg}^{2+}$, which we attributed to non-specific CBSA assembly (ESI, Fig. S1A, $\uparrow$ no cocaine). The presence of cocaine only generated an additional $7 \%$ quenching due to the absence of the $\mathrm{Mg}^{2+}$ counterion, resulting in strong repulsion between the CBSA fragments (ESI, Fig. S1A, $\dagger$ cocaine). At high concentration of $\mathrm{Mg}^{2+}(1000 \mu \mathrm{M})$, extensive CBSA assembly occurred in the absence of cocaine, producing $38 \%$ ATMND quenching, while also reducing the binding affinity of CBSA to cocaine, which only produced an additional $17 \%$ quenching upon addition of $250 \mu \mathrm{M}$ cocaine (ESI, Fig. S1A $\dagger$ ). We also varied the ATMND concentration from 50 to $1000 \mathrm{nM}$, and found that $200 \mathrm{nM}$ ATMND produced low background and high target-induced signal change (ESI, Fig. S1B $\dagger$ ). Under these optimized conditions (200 nM ATMND and $100 \mu \mathrm{M} \mathrm{Mg}^{2+}$ ), we examined the extent of target-induced CBSA assembly at different cocaine concentrations. We found a strong correlation between ATMND quenching from CBSA assembly and cocaine concentration in the range of 0 to $250 \mu \mathrm{M}$ (ESI, Fig. S2 $\dagger$ ). We characterized the binding affinity of the assembled-CBSA for ATMND by titrating different concentrations $(0-20 \mu \mathrm{M})$ of CBSA into $200 \mathrm{nM}$ ATMND in the presence of $1 \mathrm{mM}$ cocaine (ESI, Fig. S3 $\dagger$ ). The calculated $K_{\mathrm{D}}$ was $365 \mathrm{nM}$, which is consistent with the reported value for ATMND binding to AP sites. ${ }^{32}$ We subsequently demonstrated that the presence of two binding sites makes CBSA-5325 far more responsive to the presence of cocaine than split aptamers containing a single binding domain. We produced a long split aptamer (LSA) from CBSA, in which we replaced one of the binding domains with fully complementary sequences (Fig. 3A, LSA). The LSA fragments quenched $75 \%$ of ATMND fluorescence in the absence of cocaine. Addition of $250 \mu \mathrm{M}$ cocaine only induced an additional $10 \%$ signal change, indicating that most LSA fragments were stably pre-assembled even without target (Fig. 3B). In contrast, CBSA-5325 generated a large signal change ( $72 \%$ ) upon addition of $250 \mu \mathrm{M}$ cocaine because of its dual target binding domains, with far weaker background signal (9\%) without cocaine.

Specific target binding at both domains is required for target-CBSA assembly. We tested the binding affinity of 38-GC and several point-mutated derivatives using isothermal titration calorimetry (ITC) (ESI, Fig. S4†). We found that replacing an adenosine at position 22 with guanine completely impaired cocaine binding (ESI, Fig. S4, $\uparrow$ 38-GC-22G). Based on this finding, we created two CBSA mutants (CBSA-M1 and CBSA-M2) 

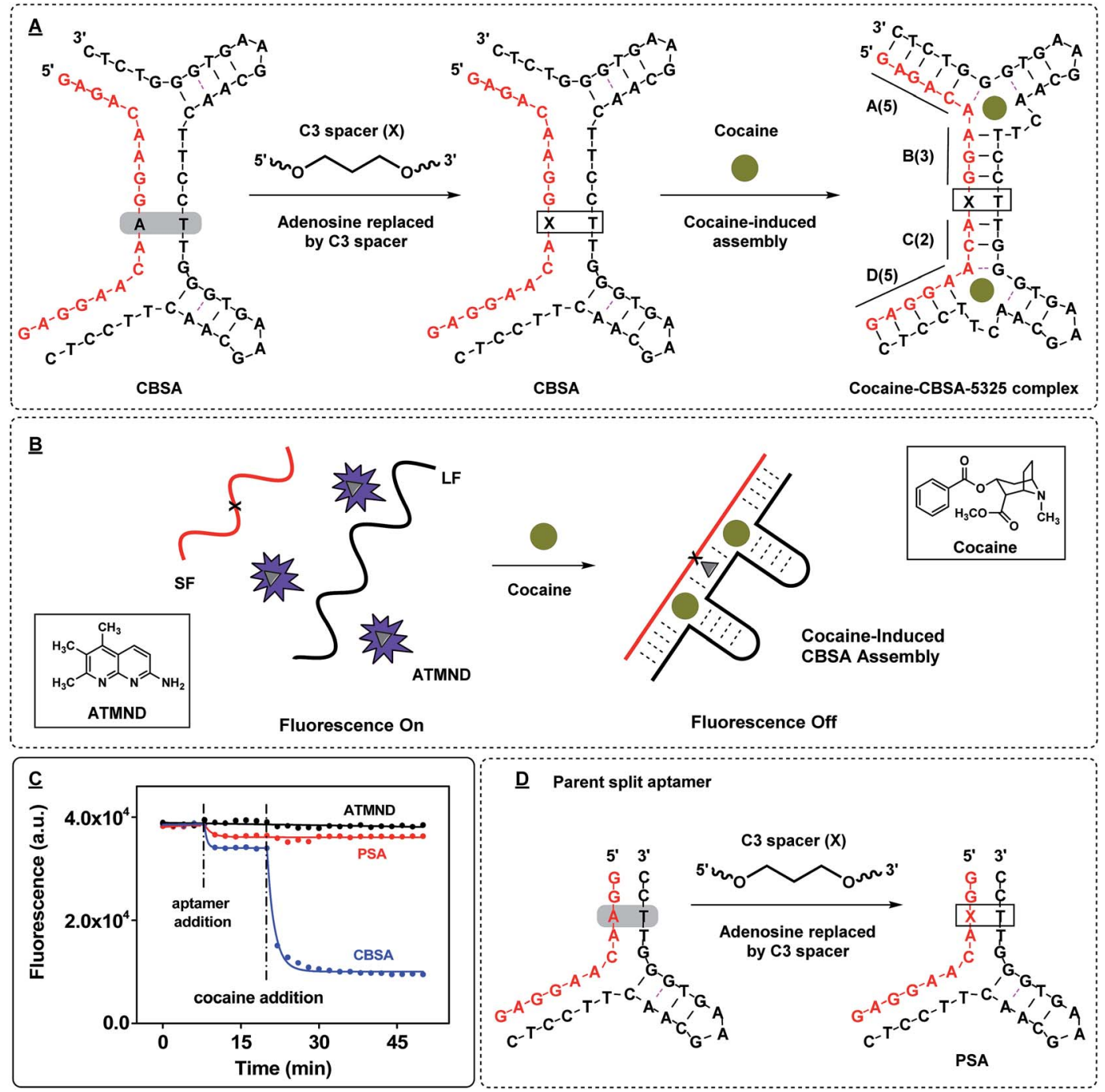

Fig. 2 ATMND as a fluorescence reporter of target-induced CBSA assembly. (A) We modified the core CBSA sequence (left) by replacing the adenosine (at position 10 from $5^{\prime}$ ) between the two binding domains of the short fragment with a C3 spacer abasic site (marked as X) to yield the final CBSA construct (middle). Upon binding cocaine, the CBSA undergoes target-induced assembly, forming a dinucleotide bulge within each three-way junction (right). The cocaine-CBSA-5325 complex features four complementary double-stranded regions (A-D) and a duplexed AP site. (B) In the absence of cocaine, LF and SF remain separated and the unbound ATMND in solution generates a strong fluorescence signal. Cocaine induces CBSA assembly, forming the duplexed AP site that binds ATMND and thereby quenches its fluorescence. (C) Time-course of ATMND quenching by cocaine-induced split aptamer assembly. (D) Sequence of the parent split aptamer (PSA) with incorporated AP site.

in which either of the two binding domains was disrupted by this single-nucleotide mutation, leaving only a single binding domain capable of binding cocaine (Fig. 3A). CBSA-M1 and CBSA-M2 were mutated at the $3^{\prime}$ - and $5^{\prime}$-binding domain of the long fragment, respectively. We tested these mutants using the same ATMND-binding assay, and found that neither CBSA-M1 nor CBSA-M2 was capable of cocaine-induced aptamer assembly, with no significant ATMND quenching observed upon addition of cocaine (Fig. 3B). These results confirmed that both target-binding domains of the CBSA were required for target-induced aptamer assembly, and thus provide strong support for a cooperative-binding-based assembly mechanism.

We hypothesized that the binding affinity of the CBSA might be enhanced by further stabilizing the target/aptamer complex with additional base-pairs, based on prior findings that longer complementary stems surrounding the three-way junction increased the aptamer's target-binding affinity. ${ }^{37}$ Thus, we increased the total number of base-pairs between the two binding domains of CBSA-5325 by adding an additional one, two or three base-pairs to generate CBSA-5335, -5435 and -5445 (ESI, Fig. S5A $\dagger$ ) and examined the extent of target-induced CBSA assembly at different cocaine concentrations $(0-50 \mu \mathrm{M})$ using our ATMND-binding assay. Our results demonstrated that the number of additional base-pairs greatly affects both binding affinity and target-induced assembly. In the absence of cocaine, the quenching of ATMND fluorescence increased as the number of base-pairs between the two CBSA binding domains increased from 5- to 8-bp (ESI, Fig. S5B $\dagger$ ). This is probably due to the 


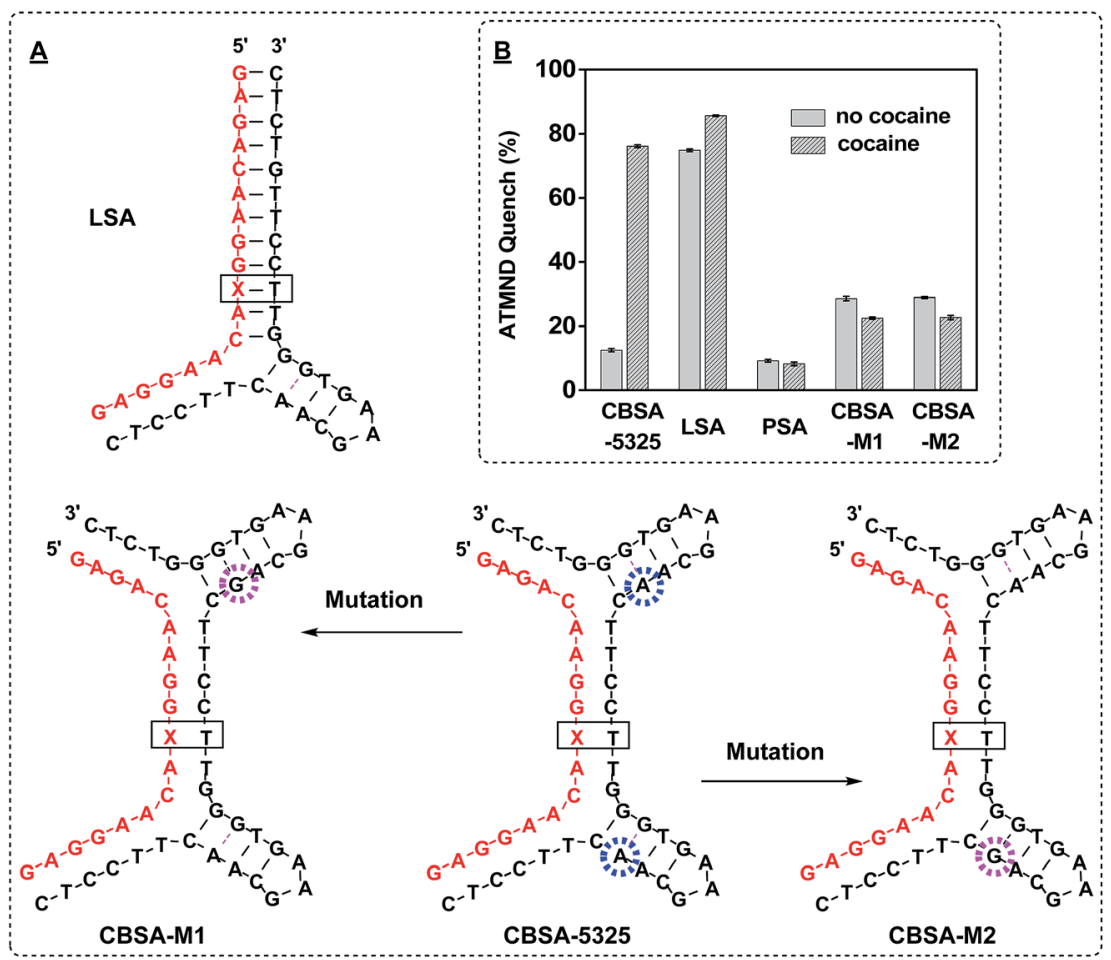

Fig. 3 Analyzing the dual binding domains of CBSA for target-induced assembly. (A) We generated multiple derivatives of CBSA-5325, including split aptamers with a single binding pocket (LSA) and a pair of point-mutants (CBSA-M1 and CBSA-M2) with sequence alterations that disrupt either of the two binding domains (pink circle). (B) ATMND quenching in the presence or absence of $250 \mu M$ cocaine. Quenching was calculated by $\left(F_{\mathrm{A}}-F\right) / F_{\mathrm{A}} \times 100 \%$, where $F_{\mathrm{A}}$ is the fluorescence of $200 \mathrm{nM}$ ATMND in $1 \times$ binding buffer alone and $F$ is the fluorescence of the ATMND-CBSA mixture with $250 \mu \mathrm{M}$ cocaine or without cocaine, respectively.

increased thermo-stability of CBSA assembly, even without target. Upon addition of cocaine, we found a strong correlation between ATMND quenching from CBSA assembly and cocaine concentration in the range of 0 to $50 \mu \mathrm{M}$ (ESI, Fig. S5C $\dagger$ ). Compared to CBSA-5325, quenching saturation occurred at lower target concentrations for the other CBSAs, in keeping with the assumption that the CBSA binding affinity can be enhanced with additional base-pairs. However, considerable background assembly was observed for CBSA-5435 and CBSA-5445 in the absence of cocaine, reducing their target-induced signal gain. We thus found that CBSA-5335 is most responsive and exhibited the most extensive target-induced CBSA assembly, and we therefore used this construct for subsequent sensor development.

We then produced fluorophore/quencher-modified derivatives of CBSA-5325 and CBSA-5335 to achieve sensitive detection of cocaine. The short fragment was modified with an IowaBlack RQ quencher at its $5^{\prime}$ terminus and a Cy5 fluorophore at its $3^{\prime}$ terminus. In the absence of cocaine, the two CBSA fragments remain separate, and the flexibility of the unbound short fragment routinely brings the fluorophore into close proximity with the quencher, resulting in very low fluorescence (Fig. 4A, left). In the presence of cocaine, the two fragments assemble to form a rigid target/aptamer structure that separates the fluorophore/ quencher pair, producing increased fluorescence (Fig. 4A, right). We used these two fluorophore/quencher-modified
CBSAs to generate calibration curves for cocaine concentrations ranging from 0-1000 $\mu \mathrm{M}$ (Fig. 4B; spectra of CBSA-5335 shown in ESI, Fig. S6†) and used the Hill equation (eqn (1)) to fit the binding curve to calculate $K_{1 / 2}$ and the Hill coefficient $\left(n_{\mathrm{H}}\right):^{30,38}$

$$
\text { Binding site occupancy } \bar{Y}=\frac{[\text { target }]^{n_{\mathrm{H}}}}{[\text { target }]^{n_{\mathrm{H}}}+\left(K_{1 / 2}\right)^{n_{\mathrm{H}}}}
$$

We determined a $K_{1 / 2}$ of $106 \mu \mathrm{M}$ with an $n_{\mathrm{H}}$ of 1.1 for CBSA5325 and a $K_{1 / 2}$ of $36 \mu \mathrm{M}$ with an $n_{\mathrm{H}}$ of 1.5 for CBSA-5335 (where $K_{1 / 2}$ represents the cocaine concentration at which half of the binding domains are occupied and $n_{\mathrm{H}}$ describes the order of binding cooperativity). ${ }^{38}$ An $n_{\mathrm{H}}$ of 1.5 clearly indicates higher cooperativity between the two binding domains of CBSA-5335. We found that the measurable LOD was $500 \mathrm{nM}(4.5 \pm 0.8 \%)$ and $50 \mathrm{nM}(4.3 \pm 0.9 \%)$ for CBSA-5325 and CBSA-5335, respectively (Fig. 4B). Clearly, the target-binding affinity of CBSA can be affected by the cooperativity of the two binding domains. Note that CBSA-5335 has the highest cocaine affinity of any split aptamer described to date, and the LOD of CBSA-5335 is more than 200-fold lower than that of a previously-described singledomain, split aptamer-based cocaine assay using a similar sensing platform $(\mathrm{LOD}=10 \mu \mathrm{M}) .{ }^{17}$

We further used ITC to characterize the binding mechanism and affinity of CBSA-5335 for its target at both sites. Recognizing that the $K_{\mathrm{D}}$ values of the aptamers were in the $\mu \mathrm{M}$ range, we set 

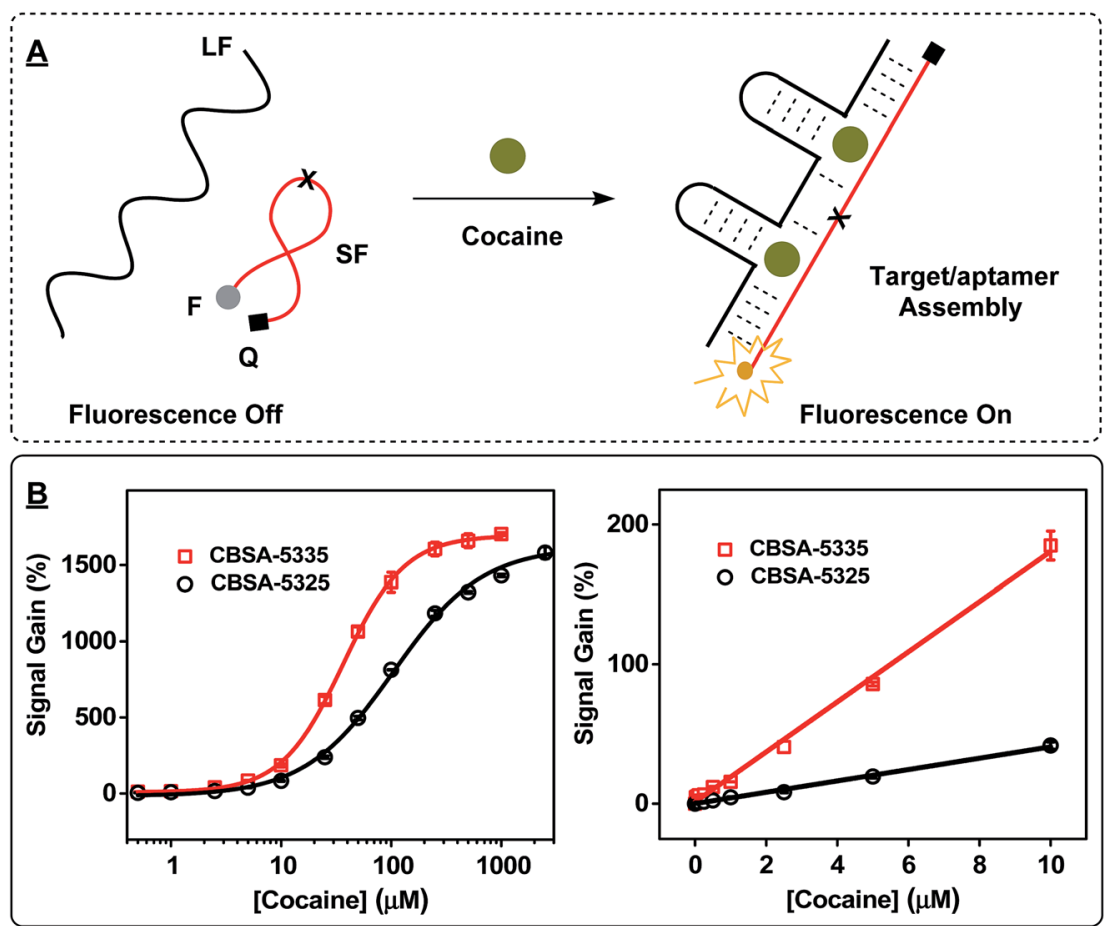

Fig. 4 Effect of target-binding affinity $\left(K_{1 / 2}\right)$ on CBSA cooperativity. (A) The working principle of the CBSA-based fluorophore/quencher assay. (B) Calibration curves for the assay at cocaine concentrations ranging from 0 to $2500 \mu \mathrm{M}$ (left). Right panel shows a linear response at $0-10 \mu \mathrm{M}$. Reactions were performed with $1 \mu \mathrm{M}$ CBSA long fragment, $1 \mu \mathrm{M}$ fluorophore/quencher-modified CBSA short fragment, and different concentrations of cocaine in $10 \mathrm{mM}$ Tris $-\mathrm{HCl}, 100 \mu \mathrm{M} \mathrm{MgCl}_{2}(\mathrm{pH} 7.4)$ at room temperature.

up a titration with a high cocaine : aptamer ratio to obtain accurate $K_{\mathrm{D}}$ values for each binding scenario (the adjacent binding pocket empty, or occupied by cocaine), where $K_{\mathrm{D}}$ represents the ligand concentration at which half of the receptor sites are occupied at equilibrium. ${ }^{39}$ The resulting twophase titration curve confirmed the interaction of cocaine with the two binding domains of CBSA-5335 (ESI, Fig. S7A $\dagger$ ). The binding stoichiometry between cocaine and CBSA was manually set as two, because previous studies have demonstrated that one cocaine-binding aptamer binds a single molecule of cocaine. $^{31}$ ITC data of CBSA-5335 were then fitted with both independent-sites and cooperative-sites models ${ }^{\mathbf{4 0}}$ with two binding sites (ESI, Fig. S7A, $\uparrow$ black broken line represented independent-sites model and red solid line represented cooperative-sites model). We observed better fitting using the cooperative-sites model, and determined that the $K_{\mathrm{D}}$ for the initial and secondary cocaine-binding events were 116 and 36 $\mu \mathrm{M}$, respectively (ESI, Fig. S7A $\dagger$ ). Based on these $K_{\mathrm{D}} \mathrm{S}$, we calculated a $K_{1 / 2}$ of $65 \mu \mathrm{M}$ and an $n_{\mathrm{H}}$ of 1.3 for CBSA-5335, ${ }^{41}$ which is comparable with the results obtained via the CBSA5335 -based fluorophore/quencher assay $\left(K_{1 / 2}=36 \mu \mathrm{M}\right.$ and $\left.n_{\mathrm{H}}=1.5\right)$. Notably, the low $K_{1 / 2}$ of CBSA-5335 represents a 56fold higher target binding affinity relative to its single-domain PSA $\left(K_{\mathrm{D}}=2 \mathrm{mM}\right.$, ESI, Fig. S7B $\left.\dagger\right)$.

We believed that the target-binding affinity of CBSA could be affected by the intrinsic binding affinity of its parent split aptamer. To address this point, we replaced a G-C pair in the $5^{\prime}$ binding domain of CBSA-5335 with a wobble G-T pair to form
CBSA-5335-GT. This alteration in stem 3 of the cocaine-binding aptamer reduces its binding affinity to cocaine ${ }^{31}$ (Fig. 5A), but we expected the resulting CBSA to still retain its cooperativity. Indeed, our fluorescence results demonstrated that both CBSAs have identical cooperativity $\left(n_{\mathrm{H}}=1.5\right)$ (Fig. 5B). However, since the intrinsic affinity of 38 -GC is 4 -fold higher than the parent split aptamer variant used for CBSA-5335-GT, CBSA-5335 yields a lower $K_{1 / 2}(33 \mu \mathrm{M})$ and better sensitivity (LOD $=50 \mathrm{nM}$ in buffer $)$ than CBSA-5335-GT $\left(K_{1 / 2}=125 \mu \mathrm{M}\right.$ and LOD $=500 \mathrm{nM}$ in buffer) (Fig. 5B).

We subsequently confirmed that our CBSA-5335-based fluorophore/quencher assay is capable of equally sensitive cocaine detection in saliva samples. The excitation wavelength for Cy5 $(648 \mathrm{~nm})$ does not induce auto-fluorescence in the saliva matrix, and thus produces minimal background fluorescence (ESI, Fig. S8†). Additionally, the high quenching efficiency of the IowaBlack RQ quencher ${ }^{42}$ allowed robust detection of cocaine at very low concentrations. To ensure that our CBSA-5335-based fluorophore/quencher assay can be reliably used in real-world (i.e., clinical or field) settings, we evaluated the assay's performance according to Scientific Working Group for Forensic Toxicology (SWGTOX) Standard Practices. ${ }^{43}$ To this end, we performed detailed experiments to investigate matrix effects, reaction time, limit of detection, interference effects and bias and precision in saliva samples.

To test the matrix effects on assay performance, we mixed eight different saliva samples collected from healthy and drugfree donors of diverse gender and ethnic backgrounds as 

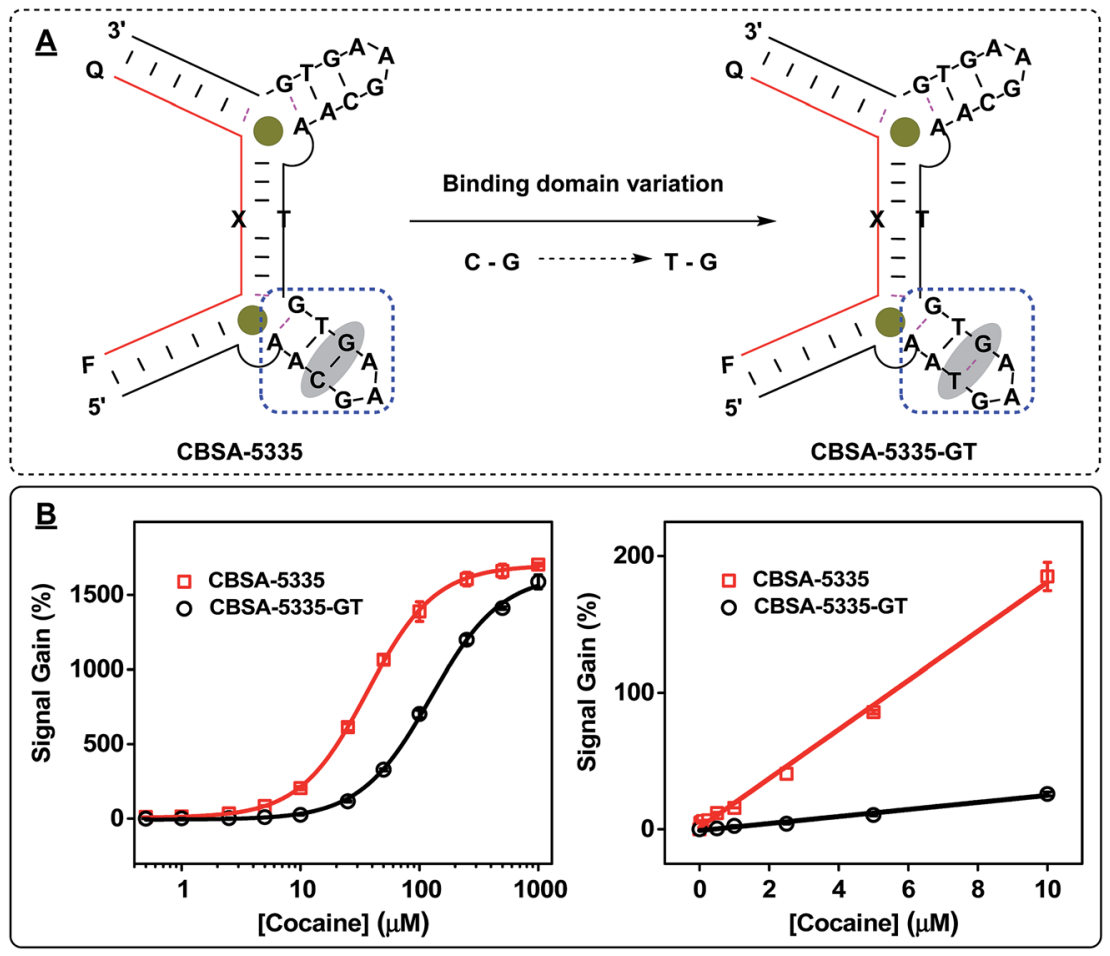

Fig. 5 Effect of the intrinsic affinity of the PSA on CBSA target-binding affinity $\left(K_{1 / 2}\right)$. (A) We generated CBSA-5335-GT by replacing a G-C basepair (highlighted) in the $5^{\prime}$ binding domain of CBSA-5335 with a G-T wobble pair. (B) Calibration curves for the assay at cocaine concentrations ranging from 0 to $1000 \mu \mathrm{M}$ after a 15 min incubation (left), with a linear response at $0-10 \mu \mathrm{M}$ (right). Experiments were performed with $1 \mu \mathrm{M} C B S A$ long fragment, $1 \mu \mathrm{M}$ fluorophore/quencher-modified CBSA short fragment, and different concentrations of cocaine in $10 \mathrm{mM}$ Tris- $\mathrm{HCl}, 100 \mu \mathrm{M}$ $\mathrm{MgCl}_{2}(\mathrm{pH} 7.4)$ at room temperature.

a pooled matrix. The pooled matrix was then spiked with different concentrations of cocaine $(0$ to $500 \mu \mathrm{M})$ and diluted with binding buffer $1: 1(50 \%)$ or $1: 9(10 \%)$ before being applied to the CBSA-5335-based fluorophore/quencher assay. Our results showed that the $10 \%$ dilution resulted in a higher signal gain with a broader dynamic range $(0-100 \mu \mathrm{M}$ in the initial saliva sample) compared to the $50 \%$ dilution $(0-25 \mu \mathrm{M}$ in the initial sample) (Fig. 6A). Additionally, the 10\% dilution compensates for variations (such as salt concentration and $\mathrm{pH}$ ) in individual saliva samples, and we therefore used $10 \%$ dilutions for subsequent experiments.

We also monitored the time course of our CBSA-5335based fluorophore/quencher assay in the $10 \%$ pooled saliva matrix. We found that the fluorescence signal greatly increased with the increase of reaction time upon the addition of 1,5 or $10 \mu \mathrm{M}$ cocaine and $85 \%$ of the maximum signal was obtained after 15 min (Fig. 6B). Clearly, the fast reaction time of our assay is suitable for on-site detection due to the rapid assembly of the cocaine-CBSA complex in saliva samples.

The CBSA-5335-based fluorophore/quencher assay also demonstrates excellent performance in target detection in complex samples. Studies have shown that cocaine concentrations are generally higher in saliva than serum within the first few hours of administration, ${ }^{44,45}$ and the European Union's Driving Under the Influence of Drugs, Alcohol and Medicines (DRUID) program identified $510 \mathrm{nM}$ as the recommended cut-off sensitivity for road-side screening of cocaine in undiluted saliva. ${ }^{44}$ To determine the sensitivity of our assay, we generated a calibration curve in $10 \%$ saliva samples, obtaining a linear range from 0 to $10 \mu \mathrm{M}$ and a measurable LOD of $50 \mathrm{nM}$ (signal gain $3.3 \pm 0.8 \%$, Fig. 7A and ESI, Fig. S9†). This suggests that, accounting for the tenfold sample dilution, our assay can meet the recommendations established by DRUID for on-site detection of cocaine, with a detectable LOD equivalent to $500 \mathrm{nM}$ in undiluted saliva. In contrast, split aptamers containing a single binding domain have previously achieved LODs of $30 \mathrm{nM}$ in $0.5 \%$ saliva, ${ }^{46} 5 \mu \mathrm{M},{ }^{47}$ and $3.8 \mu \mathrm{M}^{48}$ in $25 \%$ saliva (ESI, Table S1†). Two factors contribute to the high sensitivity of our CBSA assay. First, the low thermo-stability of the split aptamer greatly suppresses non-specific assembly of the CBSA fragments. Second, the cooperative binding from the two target-binding domains significantly increases the CBSA's affinity.

We then investigated the specificity of the CBSA-5335based fluorophore/quencher assay for cocaine versus structurally-similar and -dissimilar interferents in saliva. Benzoylecgonine (BZE), anhydroecgonine methyl ester (MEG), and cocaethylene (EC) are major structurally-similar metabolites of cocaine that are secreted into oral fluids. ${ }^{49}$ We tested our CBSA-5335-based assay with high concentrations of these metabolites as well as nicotine (NIC), since tobacco is widely used among cocaine users. We found that our CBSA assay 

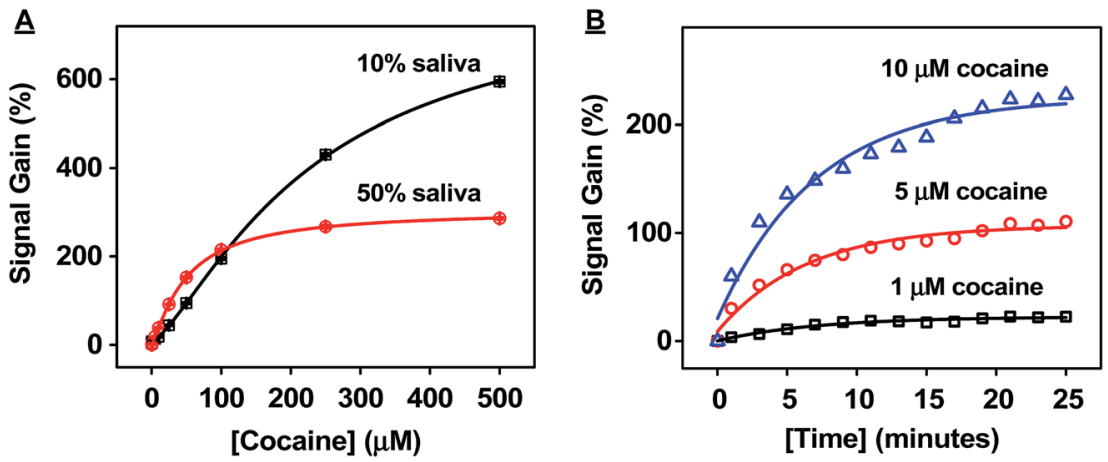

Fig. 6 Successful detection of cocaine in saliva samples with fluorophore/quencher-modified CBSA-5335. (A) Dilution effects on cocaine detection in saliva. Saliva samples spiked with cocaine were tested with CBSA-5335 at $50 \%$ or $10 \%$ dilutions. Calibration curves were constructed based on signal gain at each concentration of cocaine in the pre-dilution samples. (B) Time course of CBSA-5335 in the presence of 1,5 and $10 \mu \mathrm{M}$ cocaine in $10 \%$ pooled samples.

showed excellent cocaine specificity: our results demonstrated no measurable signal from $50 \mu \mathrm{M}$ of BZE, MEG, or NIC and only $19 \%$ and $3 \%$ cross-reactivity to $50 \mu \mathrm{M}$ and $5 \mu \mathrm{M}$ EC, respectively, in $10 \%$ saliva (Fig. $7 \mathrm{~B}$ ). The results are consistent with previously reported assays ${ }^{17,20}$ based on single-domain cocaine-binding split aptamers, demonstrating that CBSA retains excellent specificity for its target molecule in saliva. Finally, we tested the bias and precision of our CBSA assay by spiking cocaine at low, medium, and high concentrations into $10 \%$ saliva samples (final concentration of 1,5 or $10 \mu \mathrm{M}$ ) from eight different individuals (ESI, Fig. S10A $\dagger$ ). Using the pooled saliva as a standard, the average bias of signal gain obtained in these individual samples was $12.7 \%,-0.4 \%$ and $-5.8 \%$ for 1,5 and $10 \mu \mathrm{M}$ cocaine, respectively. At 1,5 and $10 \mu \mathrm{M}$, the coefficients of variation $(\mathrm{CV})$ within samples were $7.1 \%, 5.2 \%$ and $9.0 \%$, respectively, and the $\mathrm{CV}$ between runs were $7.3 \%, 5.1 \%$ and $8.5 \%$, respectively (ESI, Fig. S10B $\dagger$ ). Thus, the bias and CV were consistently below the acceptable cut-off $(20 \%)$ for drug-screening methods, ${ }^{43}$ further demonstrating the immediate feasibility of our CBSA-based assay for on-site drug screening.

\section{Conclusions}

We have demonstrated greatly improved split-aptamer-based sensing of cocaine in both buffer and saliva with a CBSA that incorporates two tandem target-binding domains into a single split aptamer. The CBSA fragments remain separate in the absence of target but specifically assemble upon addition of cocaine, and show high sensitivity to target concentration as a result of increased binding affinity caused by cooperative binding behavior. We achieved rapid, one-step detection of cocaine by simply mixing our CBSA components, which consist of a fluorophore/quencher-modified short fragment and an unmodified long fragment, with a cocaine-containing sample at room temperature. A measurable LOD of $50 \mathrm{nM}$ in buffer was achieved within 15 minutes without any signal amplification. The sensor's performance was also very robust in saliva, achieving a LOD of $50 \mathrm{nM}$ cocaine in $10 \%$ diluted saliva. Our assay also demonstrates very high target specificity, as evidenced by acceptably low cross-reactivity to common interferents, strong reproducibility, as well as a cost of $\$ 1.28$ per test, confirming the potential value of our CBSA-based assay for onsite detection of cocaine.
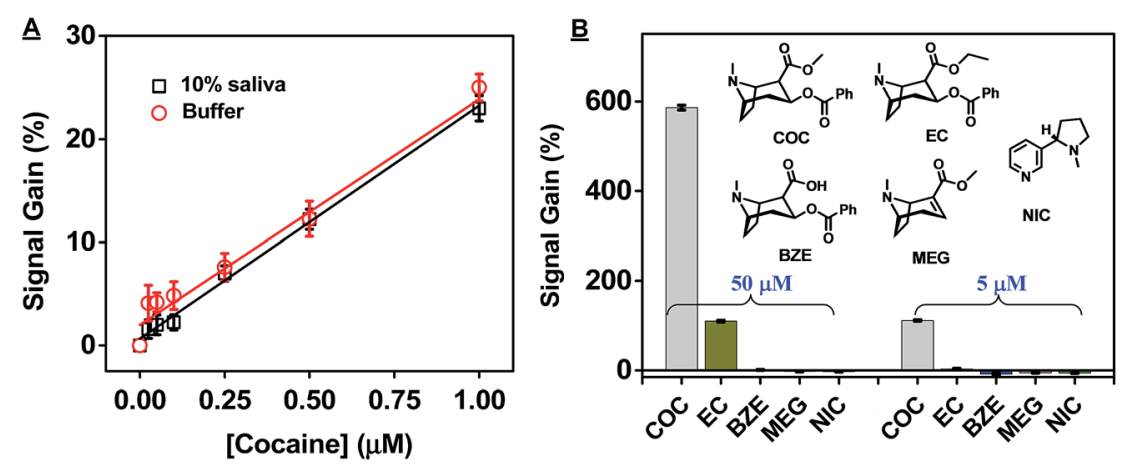

Fig. 7 High sensitivity and specificity of our CBSA-5335-based fluorophore/quencher assay for cocaine detection in saliva. (A) Calibration curve for the assay in buffer and 10\% saliva. (B) Signal gains from the CBSA assay in the presence of $50 \mu \mathrm{M}$ (left) and $5 \mu \mathrm{M}$ (right) cocaine (COC) or potential interferents including cocaethylene (EC), benzoylecgonine (BZE), anhydroecgonine methyl ester (MEG) and nicotine (NIC) in $10 \%$ saliva. Inset shows the interferent structures. Error bars show standard deviation of signal gain from three measurements at each concentration. 
Our CBSA-based assay can be generalized in terms of both targets and sensor platforms. Stojanovic et al. recently isolated new aptamers for four different steroids via heterogeneous SELEX using partially randomized DNA libraries with a predesigned, three-way-junction binding domain. ${ }^{50}$ We believe that such a selection strategy could similarly be applied for the isolation of aptamers for other drugs of abuse as well as clinically relevant targets such as small-molecule biomarkers, toxins and therapeutics. Based on Heemstra's general approach to split three-way-junction structured aptamers ${ }^{51}$ and the simplicity of engineering a CBSA from a split aptamer, it should be straightforward to develop other CBSA-based assays from the isolated aptamers for which the target-binding domain has been identified. Since many optical and electrochemical sensing strategies have been developed that employ targetinduced split aptamer assembly, ${ }^{21,52-55}$ it should be feasible to integrate our CBSA into these platforms. We also foresee the potential to improve the performance of our CBSA-based assay by employing signal amplification techniques ${ }^{53-57}$ that could further enhance its sensitivity.

\section{Experimental}

\section{Materials}

2-Amino-5,6,7-trimethyl-1,8-naphthyridine (ATMND) was purchased from Ryan Scientific and dissolved in dimethyl sulfoxide (DMSO) as a stock solution $(50 \mu \mathrm{M})$. Cocaine hydrochloride was purchased from Sigma-Aldrich. Benzoylecgonine tetrahydrate, (-)-nicotine, anhydroecgonine methyl ester and cocaethylene were purchased from Cerilliant Corporation and prepared as $50 \mathrm{mM}$ stock solutions in deionized water and stored at $4{ }^{\circ} \mathrm{C}$. Saliva samples were collected from eight healthy and drug-free volunteers (four males and four females) and centrifuged at $20000 \mathrm{rcf}$ for $15 \mathrm{~min}$, with supernatants stored at $4{ }^{\circ} \mathrm{C}$ before use. The pooled saliva matrices were prepared by mixing $1 \mathrm{~mL}$ of saliva supernatant from each donor. All DNA molecules were ordered from Integrated DNA Technologies and purified with HPLC and the sequences are listed in the ESI (Table S2 $\dagger$ ). DNA was dissolved in PCR grade water and DNA concentrations were measured with a NanoDrop 2000 (Thermo Scientific).

\section{Characterization of split aptamer assembly using ATMND}

For each ATMND-binding assay, we mixed $10 \mu \mathrm{L}$ of $10 \times$ binding buffer (100 mM Tris, $1 \mathrm{mM} \mathrm{MgCl}_{2}$, pH 7.4), $85 \mu \mathrm{L}$ of deionized water, $1 \mu \mathrm{L}$ of each split aptamer fragment (final concentration $1 \mu \mathrm{M}$ ), $1 \mu \mathrm{L}$ ATMND solution (final concentration $200 \mathrm{nM}$ ) and $2 \mu \mathrm{L}$ of cocaine at different concentrations into wells of a 96-well plate. Fluorescence intensity was measured using Tecan M1000Pro with excitation at $358 \mathrm{~nm}$ and emission at $405 \mathrm{~nm}$ at a 2 minute time interval until the fluorescence intensity was stable. Each sample was analyzed in triplicate, and the means and standard deviations were plotted. Quenching was calculated by $\left(F_{\mathrm{A}}-F\right) / F_{\mathrm{A}} \times 100 \%$, where $F_{\mathrm{A}}$ is the fluorescence of 200 nM ATMND in $1 \times$ binding buffer alone and $F$ is the fluorescence of the ATMND-CBSA mixture with or without cocaine.
Error bars show standard deviations obtained from three measurements.

\section{Isothermal titration calorimetry (ITC) experiments}

ITC experiments were performed with a MicroCal ITC200 (GE Healthcare). Cocaine and split aptamers were prepared with $1 \times$ binding buffer. The sample cell was initially loaded with $20 \mu \mathrm{M}$ of CBSA-5335 or PSA. $4 \mathrm{mM}$ of cocaine titrant was loaded into the syringe. Each experiment typically consisted of 39 successive $1 \mu \mathrm{L}$ injections after a $0.4 \mu \mathrm{L}$ purge injection with spacing of 210 seconds to a final molar ratio of $43: 1$ (cocaine : aptamer). Split-aptamer experiments were performed at $20{ }^{\circ} \mathrm{C}$. The raw data were first corrected based on the heat of dilution of cocaine, and then analyzed with the MicroCal analysis kit integrated into Origin 7 software. The titration curve of PSA was fitted with a single-site binding model and the titration curve of CBSA-5335 was fitted with independent-sites and cooperativesites models with two binding sites.

\section{Fluorophore/quencher-modified CBSA-5325, -5335, or -5335- GT for detection of cocaine}

For each fluorophore/quencher-modified CBSA-based assay, we mixed $10 \mu \mathrm{L}$ of $10 \times$ binding buffer, $83 \mu \mathrm{L}$ of deionized water, $1 \mu \mathrm{L}$ of the CBSA long fragment and $1 \mu \mathrm{L}$ of the fluorophore/ quencher-modified CBSA short fragment (final concentration 1 $\mu \mathrm{M}$ each), and $5 \mu \mathrm{L}$ of cocaine at different concentrations into wells of a 96-well plate. We measured the fluorescence intensity with a Tecan M1000Pro with excitation at $648 \mathrm{~nm}$ and emission at $668 \mathrm{~nm}$ at room temperature after $15 \mathrm{~min}$ of incubation. Each sample was analyzed in triplicate, and the means and standard deviations were plotted. The data were fitted with the Hill equation using Origin 9 software to calculate the Hill coefficient $\left(n_{\mathrm{H}}\right)$ and cocaine concentration producing half occupancy $\left(K_{1 / 2}\right)$. The signal gain was calculated by $\left(F-F_{0}\right) / F_{0} \times 100 \%$, where $F_{0}$ is the fluorescence of the CBSA mixture without cocaine and $F$ is the fluorescence of CBSA mixtures with different concentrations of cocaine. Error bars show standard deviation of signal gains from three individual measurements at each cocaine concentration.

\section{Assessing saliva matrix and dilution effects on the CBSA-5335- based fluorophore/quencher assay}

Cocaine was first spiked into the pooled saliva (FIU IRB: IRB-130320) to create artificial samples with concentrations ranging from 0.25 to $500 \mu \mathrm{M}$. Each sample was prepared by mixing $10 \mu \mathrm{L}$ of $10 \times$ binding buffer, $1 \mu \mathrm{L}$ of CBSA long fragment and $1 \mu \mathrm{L}$ of fluorophore/quencher-modified CBSA short fragment (final concentration $1 \mu \mathrm{M}$ each) into wells of a 96-well plate. 10 or $50 \mu \mathrm{L}$ of sample for each cocaine concentration was added into the well to analyze the $10 \%$ or $50 \%$ saliva matrices and deionized water was added to bring each well's volume to $100 \mu \mathrm{L}$. Fluorescence intensity with excitation at $648 \mathrm{~nm}$ and emission from 655-850 $\mathrm{nm}$ was scanned with a Tecan M1000Pro at room temperature after $15 \mathrm{~min}$ of incubation. Each sample was analyzed in triplicate, and the mean and standard deviation of the signal gain at different cocaine concentrations were plotted. 
Determining the sensitivity and dynamic range of CBSA-5335based fluorophore/quencher assay in saliva

For cocaine detection in $10 \%$ saliva, we combined $5 \mu \mathrm{L}$ of cocaine in solutions of concentrations ranging from 0.001 to 10 $\mu \mathrm{M}$ with $10 \mu \mathrm{L}$ of pooled saliva, $10 \mu \mathrm{L}$ of $10 \times$ binding buffer, $1 \mu \mathrm{L}$ of CBSA long fragment, $1 \mu \mathrm{L}$ of fluorophore/quenchermodified CBSA short fragment (final concentration $1 \mu \mathrm{M}$ each), and $73 \mu \mathrm{L}$ of deionized water into wells of a 96-well plate. Fluorescence intensity with excitation at $648 \mathrm{~nm}$ and emission at $668 \mathrm{~nm}$ was measured with a Tecan M1000Pro at room temperature after $15 \mathrm{~min}$ of incubation. Each sample was analyzed in triplicate, and the mean and standard deviation of the signal gain at different cocaine concentrations were plotted. A control calibration curve in buffer was also generated with cocaine concentrations ranging from 0.001 to $10 \mu \mathrm{M}$ as described above. The signal gain was calculated by $\left(F-F_{0}\right) / F_{0} \times$ $100 \%$, where $F_{0}$ is the fluorescence without cocaine and $F$ is the fluorescence with different concentrations of cocaine. Error bars show standard deviations from three measurements. The measurable LOD was determined using the lowest non-zero calibrator concentration according to the Scientific Working Group for Forensic Toxicology (SWGTOX) Standard Practices for Method Validation in Forensic Toxicology. ${ }^{43}$ In all cases, the calibrators (cocaine standard concentration) were analyzed over three runs, and the lowest concentration achieving mean signal gain higher than 3.3 times the standard deviation was defined as the measurable LOD.

\section{Determining the target specificity of CBSA-5335-based fluorophore/quencher assay in saliva}

The CBSA-5335 fluorophore/quencher assay was performed as described above with cocaine, cocaethylene, benzoylecgonine, anhydroecgonine methyl ester or nicotine at concentrations of 5 or $50 \mu \mathrm{M}$ in $10 \%$ saliva. Each sample was analyzed in triplicate and the mean and standard deviation of the signal gain at different cocaine concentrations were plotted. The cross-reactivity of each analyte at each concentration was calculated as a percentage based on $\mathrm{Sig}_{\mathrm{ANA}} / \mathrm{Sig}_{\mathrm{COC}} \times 100 \%$, where $\mathrm{Sig}_{\mathrm{ANA}}$ is the signal gain achieved by a given interferent and Sig $_{\mathrm{COC}}$ is the signal gain achieved by cocaine at the same concentration.

\section{Determining the precision and bias of CBSA-5335-based fluorophore/quencher assay in saliva}

The CBSA-5335 fluorophore/quencher assay was performed as described above in $10 \%$ diluted saliva matrices collected from eight different donors, $10 \%$ diluted pooled saliva matrices and binding buffer. We performed six measurements of samples containing final cocaine concentrations of $0,1,5$ and $10 \mu \mathrm{M}$, and plotted the mean and standard deviation of the signal gain at different cocaine concentrations. The bias of each cocaine concentration was calculated as $\left(\right.$ mean $_{\text {sam }}-$ mean $\left._{\text {pool }}\right) /$

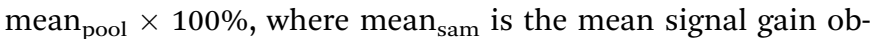
tained in $10 \%$ saliva matrices collected from different donors, and mean $_{\text {pool }}$ is the mean signal gain obtained in the $10 \%$ pooled saliva matrix. The precision within samples or between runs at different cocaine concentrations was calculated by performing a one-way ANOVA test with the measurement number (6) as the grouping variable. Within-sample precision at each cocaine level was calculated as $\sqrt{\mathrm{MS}_{\mathrm{wg}}} /$ mean $_{\mathrm{sam}} \times 100 \%$, where $\mathrm{MS}_{\mathrm{wg}}$ is the within-group mean square obtained from the ANOVA table, and mean $n_{\text {sam }}$ is the mean of signal gains obtained in $10 \%$ saliva matrices collected from different donors. Between-run precision at each cocaine level was calculated as $\sqrt{\frac{\mathrm{MS}_{\mathrm{bg}}+(n-1) \times \mathrm{MS}_{\mathrm{wg}}}{n}} / \operatorname{mean}_{\mathrm{sam}} \times 100 \%$, where $\mathrm{MS}_{\mathrm{bg}}$ is the between-group mean square obtained from the ANOVA table, and $n$ is the total number of measurements $(n=6)$.

\section{Conflict of interest}

The authors declare no competing financial interest.

\section{Acknowledgements}

This work was supported by Awards No. 2013-DN-BX-K032 and No. 2015-R2-CX-0034 awarded by the National Institute of Justice, Office of Justice Programs, U.S. Department of Justice. We greatly thank Anna Simon for her help with the calculation of $K_{1 / 2}$ using the dissociation constants obtained from ITC.

\section{Notes and references}

1 A. D. Ellington and J. W. Szostak, Nature, 1990, 346, 818-822.

2 C. Tuerk and L. Gold, Science, 1990, 249, 505-510.

3 J. F. Lee, J. R. Hesselberth, L. A. Meyers and A. D. Ellington, Nucleic Acids Res., 2004, 32, D95-D100.

4 V. J. B. Ruigrok, M. Levisson, M. H. M. Eppink, H. Smidt and J. van der Oost, Biochem. J., 2011, 436, 1-13.

5 S. D. Jayasena, Clin. Chem., 1999, 45, 1628-1650.

6 W. Mok and Y. F. Li, Sensors, 2008, 8, 7050-7084.

7 M. N. Stojanovic, P. de Prada and D. W. Landry, J. Am. Chem. Soc., 2001, 123, 4928-4931.

8 K. A. Yang, M. Barbu, M. Halim, P. Pallavi, B. Kim, D. M. Kolpashchikov, S. Pecic, S. Taylor, T. S. Worgall and M. N. Stojanovic, Nat. Chem., 2014, 6, 1003-1008.

9 L. C. Bock, L. C. Griffin, J. A. Latham, E. H. Vermaas and J. J. Toole, Nature, 1992, 355, 564-566.

10 D. E. Huizenga and J. W. Szostak, Biochemistry, 1995, 34, 656-665.

11 T. Mairal, V. C. Ozalp, P. Lozano Sánchez, M. Mir, I. Katakis and C. K. O'Sullivan, Anal. Bioanal. Chem., 2008, 390, 9891007.

12 J. H. Lee, M. V. Yigit, D. Mazumdar and Y. Lu, Adv. Drug Delivery Rev., 2010, 62, 592-605.

13 E. J. Cho, J. W. Lee and A. D. Ellington, Annu. Rev. Anal. Chem., 2009, 2, 241-264.

14 M. McKeague and M. C. DeRosa, J. Nucleic Acids, 2012, 748913.

15 M. N. Stojanovic and D. W. Landry, J. Am. Chem. Soc., 2002, 124, 9678-9679. 
16 B. R. Baker, R. Y. Lai, M. S. Wood, E. H. Doctor, A. J. Heeger and K. W. Plaxco, J. Am. Chem. Soc., 2006, 128, 3138-3139.

17 M. N. Stojanovic, P. de Prada and D. W. Landry, J. Am. Chem. Soc., 2000, 122, 11547-11548.

18 R. X. Zou, X. H. Lou, H. C. Ou, Y. Zhang, W. J. Wang, M. Yuan, M. Guan, Z. F. Luo and Y. Y. Liu, RSC Adv., 2012, 2, 4636-4638.

19 J. Nie, Y. Deng, Q. P. Deng, D. W. Zhang, Y. L. Zhou and X. X. Zhang, Talanta, 2013, 106, 309-314.

20 A. K. Sharma and J. M. Heemstra, J. Am. Chem. Soc., 2011, 133, 12426-12429.

21 A. K. Sharma, A. D. Kent and J. M. Heemstra, Anal. Chem., 2012, 84, 6104-6109.

22 M. F. Perutz, A. J. Wilkinson, M. Paoli and G. G. Dodson, Annu. Rev. Biophys. Biomol. Struct., 1998, 27, 1-34.

23 T. Meyer, D. Holowka and L. Stryer, Science, 1988, 240, 653656.

24 T. Krell, W. Terán, O. L. Mayorga, G. Rivas, M. Jiménez, C. Daniels, A. J. Molina-Henares, M. Martínez-Bueno, M. T. Gallegos and J. L. Ramos, J. Mol. Biol., 2007, 369, 1188-1199.

25 D. Bray, Nature, 1995, 376, 307-312.

26 M. Mandal, M. Lee, J. E. Barrick, Z. Weinberg, G. M. Emilsson, W. L. Ruzzo and R. R. Breaker, Science, 2004, 306, 275-279.

27 N. Sudarsan, M. C. Hammond, K. F. Block, R. Welz, J. E. Barrick, A. Roth and R. R. Breaker, Science, 2006, 314, 300-304.

28 A. M. Jose, G. A. Soukup and R. R. Breaker, Nucleic Acids Res., 2001, 29, 1631-1637.

29 A. J. Simon, A. Vallée-Bélisle, F. Ricci, H. M. Watkins and K. W. Plaxco, Angew. Chem., Int. Ed., 2014, 53, 9471-9475.

30 A. J. Simon, A. Vallée-Bélisle, F. Ricci and K. W. Plaxco, Proc. Natl. Acad. Sci. U. S. A., 2014, 111, 15048-15053.

31 D. Roncancio, H. X. Yu, X. W. Xu, S. Wu, R. Liu, J. Debord, X. H. Lou and Y. Xiao, Anal. Chem., 2014, 86, 11100-11106.

32 Y. Sato, T. Kageyama, S. Nishizawa and N. Teramae, Anal. Sci., 2013, 29, 15-19.

33 Y. Sato, S. Nishizawa, K. Yoshimoto, T. Seino, T. Ichihashi, K. Morita and N. Teramae, Nucleic Acids Res., 2009, 37, 1411-1422.

34 M. A. D. Neves, O. Reinstein, M. Saad and P. E. Johnson, Biophys. Chem., 2010, 153, 9-16.

35 O. Reinstein, M. Yoo, C. Han, T. Palmo, S. A. Beckham, M. C. J. Wilce and P. E. Johnson, Biochemistry, 2013, 52, 8652-8662.
36 R. Owczarzy, B. G. Moreira, Y. You, M. A. Behlke and J. A. Walder, Biochemistry, 2008, 47, 5336-5353.

37 M. A. D. Neves, O. Reinstein and P. E. Johnson, Biochemistry, 2010, 49, 8478-8487.

38 A. Hill, J. Physiol., 1910, 40, iv-vii.

39 W. B. Turnbull and A. H. Daranas, J. Am. Chem. Soc., 2003, 125, 14859-14866.

40 L. A. Freiburger, K. Auclair and A. K. Mittermaier, ChemBioChem, 2009, 10, 2871-2873.

41 F. W. Dahlquist, The meaning of scatchard and hill plots, in Enzyme Structure Part F, Methods in Enzymology, ed. C. H. W. Hirs and S. N. Timasheff, Academic Press, Cambridge, MA, 1978, vol. 48, pp. 270-299.

42 M. K. Johansson, Methods Mol. Biol., 2006, 335, 17-29.

43 Scientific Working Group for Forensic Toxicology (SWGTOX), Standard Practices for Method Validation in Forensic Toxicology, J. Anal. Toxicol., 2013, 37, 452-474.

44 H. Gjerde, K. Langel, D. Favretto and A. G. Verstraete, J. Anal. Toxicol., 2014, 38, 92-98.

45 W. Schramm, P. A. Craig, R. H. Smith and G. E. Berger, Clin. Chem., 1993, 39, 481-487.

46 D. L. Ma, M. D. Wang, B. Y. He, C. Yang, W. H. Wang and C. H. Leung, ACS Appl. Mater. Interfaces, 2015, 7, 1906019067.

47 Y. Shi, H. C. Dai, Y. J. Sun, J. T. Hu, P. J. Ni and Z. Li, Analyst, 2013, 138, 7152-7156.

48 Y. Du, C. G. Chen, J. Y. Yin, B. L. Li, M. Zhou, S. J. Dong and E. K. Wang, Anal. Chem., 2010, 82, 1556-1563.

49 E. J. Cone, M. Hillsgrove and W. D. Darwin, Clin. Chem., 1994, 40, 1299-1305.

50 K. A. Yang, R. J. Pei, D. Stefanovic and M. N. Stojanovic, J. Am. Chem. Soc., 2012, 134, 1642-1647.

51 A. D. Kent, N. G. Spiropulos and J. M. Heemstra, Anal. Chem., 2013, 85, 9916-9923.

52 R. Freeman, E. Sharon, R. Tel-Vered and I. Willner, J. Am. Chem. Soc., 2009, 131, 5028-5029.

53 C. H. Lu, F. Wang and I. Willner, Chem. Sci., 2012, 3, 2616.

54 X. Q. Liu, R. Freeman and I. Willner, Chem.-Eur. J., 2012, 18, 2207-2211.

55 Q. Li, Y. D. Wang, G. L. Shen, H. Tang, R. Q. Yu and J. H. Jiang, Chem. Commun., 2015, 51, 4196-4199.

56 S. Y. Niu, X. F. Lou, Y. Jiang and J. H. Lin, Anal. Lett., 2012, 45, 1919-1927.

57 Z. Zhu, C. Wu, H. Liu, Y. Zou, X. Zhang, H. Kang, C. J. Yang and W. Tan, Angew. Chem., Int. Ed., 2010, 49, 1052-1056. 